be mapped out ; the impulse was indistinct; the sounds were natural ; the lungs were normal, except for slight emphysema. The abdomen was much distended, of spherical form, rising three or four inches above the level of the sternum, and measured forty-seven and a half inches in circumference at the umbilicus. Fluctuation was distinct; the superficial abdominal veins were somewhat distended. The liver.dulness appeared to reach the lower border of the fourth rib, in the right mammary line. Neither liver nor spleen could be felt in the abdomen. There was slight pitting on pressure over the legs. The urine was scanty, acid, of specific gravity 1,012, free from albumen. The temperature, pulse, and respiration were normal. The abdomen was directed to be bandaged with a wide flannel roller. She was ordered to take mistura rubra, one ounce, three times a-day.

November I 5 th. The ascites was considerably diminished. The abdominal walls were quite flaccid and thin. The liver-dulness did not extend two fingers-breadth in the right mammary line. The general condition was improved.

December 6th. The abdomen was greatly diminished in size; it was flaccid and pendulous. There was no distinct fluctuation wave now, but there was dulness in both flanks, whilst the summit of the abdomen was resonant. There was still slight pitting over the feet and legs. She was directed to continue the abdominal pressure.

December 2oth. The patient was now up; the abdomen had not enlarged since she had left her bed. There was no œdema of the feet. She still had the kidney look observed on admission. For the last two months, she had passed, on an average, sixty-five ounces of urine daily, of pale colour, specific gravity varying between I, OIO and I,OI 5, and, though repeatedly examined, it had never contained albumen. The patient was now allowed to go home and attend as an out-patient. Whilst she continued under observation there was no recurrence of the ascites. The only medicine she took throughout her residence in the hospital was the mistura rubra of the London Hospital Pharmacopaia (burnt sugar and water).

It will be seen that in the first case alluded to the ascites was very great, and the vital condition of the patient brought very low from malassimilation. The appearance of the leg, with the absence of any history of intemperance (the latter not a very valuable indication in the case of a woman, it is true), suggested the possibility of syphilis causing the disease of liver which gives rise to the ascites. Without adopting this view, I thought it right to give the patient the advantage of the doubt, and accordingly administered iodide of potassium until the patient was fully under its influence. The ascites certainly diminished during the time the patient was taking the drug, but the diminution continued, it will be observed, when it was replaced by some quinin and iron, given as a general tonic, and was, no doubt, due to the rest in bed. I cannot ascribe any specific action to the iodide of potassium, therefore, and the patient never subsequently had any symptoms suggestive even of syphilis. Between March and May, it will be noticed, the patient made no progress. Her dropsy remained stationary. She passed at this time about forty ounces of urine daily. The idea suggested itself to me, as it had often previously, that the absorption which took place when the patient first came under treatment was due in great measure to the pressure of the greatly distended, tense, abdominal walls opening up the collateral channels of the portal circulation-the previously existing communications between the branches of the portal vein and the tributaries of the superior and inferior venæ cavæ, and the new system of vessels developed in the augmented connective tissue of the liver, and in the adhesions formed by this organ.* When a certain amount of fluid had become absorbed, the abdominal walls became flaccid, and no longer exercised pressure on the portal system sufficient to drive the blood throagh devious and narrow conduits. It occurred to me to imitate the natural process by which absorption had, it seemed, been brought about in the first instance, and to the failure of which the arrest of the absorption appeared attributable. Hence I endeavoured by a bandage to convert the lax yielding abdominal wall into a tense elastic and resisting one. The employment of a properly constructed abdominal support was only a more convenient and efficacious method of applying the necessary pressure. The result was highly encouraging. The bandage and support certainly squeezed the fluid out of the peritoneal cavity, and it was carried out of the body, without loss to the system of any albumen, by means of the kidneys. The result was a cure of the portal obstruction. It is now nearly three years since the patient left the hospital, and I have had her frequently under my observation. There has been absolutely no recurrence of the ascites. Equally satisfactory was the effect of the same treatment in the second case I have given-a case from its

* Frerichs, Disease of the Liver (New Sydenham Society's translation), vol. ii, page 29 . antecedents far from a promising one-and here the mechanical treatment was uncomplicated by the action of drugs. I may add I have had as good results in other cases.

Hitherto I have not tried abdominal compression in increasing ascitic effusions. It has seemed to me that the abdominal walls offered as much opposition to the pouring out of fluid as it would be safe to call into play, and that a greater strain on the vessels might lead to their rupture-effects we not unfrequently witness in cirrhosis, as hæmatemesis and hæmorrhage from the bowels. It is, however, quite possible that the obstacle offered to the effusion from the portal veins may be safely augmented to such a degree as to force open the anastomoses of the portal vein, and, by thus effectually carrying off the blood brought to the liver, to cure the disease. I use the word cure advisedly, as fibrosis of the liver in the main kills the patient by the secondary effects of the mechanical obstruction of the portal circulation. A person can get along, by management, with half a liver. In contraction of the liver, if the blood which cannot traverse the organ be efficiently carried off by other routes, and is not lost to the system, enough blood may yet pass through the liver to enable this large gland to perform its varied and important functions for all ordinary needs of the body. So far as I know, the plan of abdominal compression has not been recommended for cases of ascites, though I make no claim for its novelty. It has proved useful in my hands, and, I believe, will be found serviceable in causing or accelerating the absorption of an ascites, alone or in conjunction with other methods of treatment.

\section{TWO CASES OF POISONING BY PHOSPHORUS.}

\section{By FREDERICK W. WILLMORE, M.R.C.S.Eng., Walsall.}

THE recent case of death from this poison, narrated in the JOURNAL by Surgeon-Major Martin, induces me to record two of a similar nature which have been lately under my own observation, and which present several features of interest. Two young girls, L. C. and M. H., aged respectively sixteen and fourteen years, took between them, on the evening of March 25th ult., a small potful of phosphorus-paste, containing about half-an-ounce. Vomiting, with abdominal pain, rapidly came on, yet no advice was sought for until the afternoon of Friday the 29th. I was then asked to see the sufferers, being told "that they had taken poison on Monday, in consequence of slighted affection, and that they were still lingering". In compliance with this quaint request, I at once saw the patients. Both presented symptoms of grave depression; the vomiting still recurred occasionally; there was no diarrhœa, but the whole abdominal region was greatly distended, painful, and tender. The breath and vomited matter smelt strongly of the poison, and the skin and conjunctivæ were both deeply yellow. Severe frontal headache was complained of, and the act of swallowing was attended with considerable difficulty and pain. The pulse was thready and easily compressed-in the elder girl 135 ; in the younger 98 . From the first, the case of the former patient gave most cause for anxiety, and I ascertained that she had taken the larger amount of the poison. A small quantity of food and mucus, obtained from the stomach of M. H., and submitted to Mitscherlich's test, gave distinct evidence of the presence of phosphorus. The bowels having been gently cleared, small doses of morphia were given, with the effect of relieving the pain and vomiting. The case of L. C., however, rapidly became worse, the prostration more marked, and drowsiness and convulsions terminated her life on March 3 rst, eight days after taking her fatal meal.

On Tuesday, April 2nd, fifty-six hours after death, I made a careful examination of the body, of which the following is an abridged report. The stomach and bowels were greatly distended with flatus; the inner coat showing, over nearly its whole area, traces of recent inflammation, but destitute of any erosion. In the stomach itself was found a small quantity of black grumous material, with a distinct odour of phosphorus. The liver, though normal in size, presented changes of a striking character. It was of a dull yellow colour, its borders quite black, the dark rim varying from the eighth of an inch to nearly an inch in depth. Its under surface also presented black patches, contrasting strangely with the yellow body of the organ. This dark appearance seemed limited to the capsule, and did not extend to the parenchyma. The tissue itself was abnormally yellow, soft, and friable, and under the microscope its ordinary characters were quite in. distinguishable. The gall-bladder was almost empty. 'The spleen contained a small cystic tumour, but was otherwise of the usual size and appearance. The cerebral membranes, and more especially the pia mater, were deeply hyperæmic, and the arachnoidal space was filled with a considerable amount of effused fluid. The brain-substance was 
softened, and the ventricles were distended with effusion. No morbid appearances were found either in the kidneys or bladder. This latter contained nearly a pint of limpid urine. All the remaining organs were healthy; the mouth and fauces not discoloured; nor was there any trace of ecchymosis.

The girl who survives yet maintains her yellow colour, with furred tongue and scanty excretion of urine; but in other respects she daily improves, and I hope will eventually recover. I may observe that she has been treated throughout with turpentine, and, in my judgment, with marked and continued relief.

Without wishing to comment unduly upon these cases, I may yet venture to remark one or two salient points in their history. The subacute symptoms, persisting for a space of nearly five days without materially alarming the relatives, are worthy of notice. Neither the pain nor the vomiting were of a severe nature ; the bowels acted in an usual manner; and each of the patients, from time to time, took small quantities of support. Phosphorus is such an insidious and deadly poison, and in the form of paste is so accessible to the thoughtless and impulsive, that we may expect a recurrence of cases like the present. The symptoms produced by even a small dose of it are varied, and certainly not easily distinguishable; and it is only with the desire of adding some little corroborative testimony to Dr. Martin's interesting and instructive case that I have ventured to offer these few notes.

\section{REPORTS}

\section{MEDICAL AND SURGICAL PRACTICE IN THE HOSPITALS AND ASYLUMS OF GREAT BRITAIN AND IRELAND.}

\section{ST. BARTHOLOMEW'S HOSPITAL.}

DR. MATTHEWS DUNCAN'S WARDS.

FOR the notes of the following case we are indebted to Mr. W. E. Husbann, Resident Physician.

Fibroid Tiumour of the Uterus proving fatal by Pressure on the Ureters. - A. S. was admitted to the hospital on January 14th, with the following history. She had been married eleven years, but had never been pregnant. The catamenia commenced at twenty-one years of age. The last period commenced nine weeks before admission; it was very profuse, and accompanied by pain in the lumbar region and abdomen; it lasted thirteen days. The periods had generally been irregular; formerly, the intervals were frequently from four to six months. Two years ago, they became more regular, a scanty show appearing every three weeks. Six months later, the periods became profuse, with only a fortnight's interval. Latterly, there had again been a longer interval-four or five weeks; and the loss had been in. considerable.

On admission, she complained of a stabbing pain in the lower part of the abdomen, especially in the right flank, shooting down the thighs, and coming on every few hours. The cause of this pain was not suspected during life; but the necropsy showed that it was probably due to compression of the ureters by the tumour. She also complained of loss of appetite, constipation, and painful micturition, with great flow of water. The urine, when examined, was almost colourless, reaction acid, specific gravity 1003; it was free from albumen.

On examination, the abdomen was seen to be very prominent, semiglobose in shape, and occupied by a hard dense mass said to be of twelve months' duration. The most prominent point was midway between the umbilicus and symphysis pubis. The abdomen measured in girth 32 inches. The other measurements were as follows : from the navel to the ensiform cartilage, $7^{1 / 2}$ inches; to the symphysis pubis, $83 / 4$ inches; to the right superior iliac spine, $71 / 4$ inches; to the left superior iliac spine, $7 \frac{1}{4}$ inches. The whole of the prominent portion of the tumour was very hard and elastic. There was dulness on percussion over the mass up to one inch above the umbilicus, but no impairment of resonance elsewhere. Per vaginam, the true pelvis was found to be almost entirely occupied by a hardness which could be identified with the abdominal tumour above described. The vagina was natural, and contained a little white discharge. The sound passed easily into the uterus and to the left to the extent of six inches and a half. There was tenderness on both sides of the uterus, especially on the left.

Dr. Duncan thought that the pain might be diminished by relieving the great tension in the neck of the womb. The os was slightly open and very much on the stretch, the tumour growing down into its lip on one side. He therefore incised the os with a pair of scissors, hoping to relieve the tension and pain, and to reduce the hremorrhage, as in these cases is often found to follow the opening up of the neck. Two days after the operation, in the evening, a severe flooding suddenly came on, preceded for a few minutes by a sharp pain. The patient lost more than a quart of blood, the lips becoming completely blanched, and the radial pulse being imperceptible for a few moments. The flooding ceased as suddenly as it came on, and did not return till two days later, when in the middle of the day it commenced again, but was soon arrested by plugging the vagina. During the next eight days, she was troubled with uncontrollable vomiting, and no medicine had any effect in diminishing it. At the end of this period, she began to have frequent muscular twitchings, and later two actual convulsive attacks. She also had a limited herpetic eruption upon the perinæum and labia; nowhere else. This came on suddenly, and disappeared almost as quickly. The patient died on February $3^{\text {rd, the twitchings }}$ having gradually spread upwards, at last involving the face.

Necropsy. - The stomach was distended. There was no recent peritonitis ; but there was evidence of old peritonitis, the upper surface of the liver and spleen being adherent to the diaphragm; but these organs appeared healthy. The right kidney was small and wasted; its capsule was easily removed, leaving the surface smooth, pale, mottled with a few blood-vessels; the cortex was diminished and white the pyramids were pink; the ureter was much dilated and tortuous, nearly large enough to admit the finger. The left kidney and ureter were in the same state as the right. The kidneys weighed eleven ounces. There were no clots in the inferior vena cava or spermatic veins. At the junction of the left internal iliac vein with the external was a large fibrinous clot, not completely organised, but filling up the cavity of the vessel. On following the branches of the internal iliac vein, a branch was found coming from the spine and emptying into the obstructed vessel; it was completely blocked by a nearly organised clot. Filling up nearly the whole of the pelvis, was a large fibroid tumour weighing four pounds ten ounces, and pushing the bladder over to the left; it was covered by a thin muscular capsule. There was a large vein on the right side of the tumour, dilated, tortuous, and empty. The tumour was hard and pale, no blood-vessel being seen in its substance. The cavity of the uterus was displaced to the left; it was very elongated and dilated, and very pale ; the cervix was obliterated. The right ovary was very pale. The mucous membrane of the bladder was very pale. It appeared that the tumour had been jammed into the pelvis and compressed the ureters; these, becoming greatly dilated and tortuous, irritated the kidneys, and their substance became diseased. The nervous phenomena referred to were uræmic, due to compression of the ureters. The urine, when examined, had a low specific gravity, but was free from albumen. As it was passed involuntarily, it could not be re-examined.

Operation for Removal of an Uterine Polypus. - A woman, the subject of uterine polypus, came to the hospital for its removal. A large mass was felt projecting into the vagina, but neither the finger nor the uterine sound could be passed beyond the tumour to ascertain exactly its connection. Dr. Duncan referred to the importance of exact diagnosis before operation in these cases, instances having occurred in which an inverted uterus had been cut off under the impression that it was a polypus.

The patient being placed in the lithotomy position, the tumour was seized with a volsella, which was then brought downwards and forwards against the pubis; a second volsella was then used to catch the tumour at its posterior part and drag it near to the orifice. Digital examination revealed a distinct and firm pedicle, half an inch thick and about an inch and a half long, which passed up into the uterus, and the sound passed without difficulty the normal depth into the uterus. A strong pair of curved scissors was used to divide the pedicle ; the operation was quite painless. The uterus itself appeared perfectly healthy, and contracted immediately after the operation, so that the stump of the pedicle could no longer be felt.

On examining the tumour removed, the pedicle, which had been cut at least an inch from its extremity, no longer projected from the tumour, but had shrunk into its mass. The tumour measured three inches in diameter ; it was nodular and inflamed upon the surface, a condition not usually seen ; in structure it appeared fibroid.

Parametritic Induration. - In seeking evidence by physical examination upon which to found a diagnosis in another case, Dr. Duncan commenced by examining the abdomen. Nothing abnormal was felt, but there was some fulness in the hypogastrium, about the region of the brim of the pelvis ; in this locality the abdominal wall could not be depressed as easily as should be. In this case, as is usual in well-formed women, the promontory of the sacrum could be easily felt lying but a 